\title{
Acupuncture for Chronic Knee Pain A Randomized Clinical Trial
}

\begin{abstract}
Rana S. Hinman, PhD; Paul McCrory, PhD; Marie Pirotta, PhD; lan Relf, MSc; Andrew Forbes, PhD; Kay M. Crossley, PhD; Elizabeth Williamson, PhD; Mary Kyriakides, BAppSc; Kitty Novy, BNurs;

Ben R. Metcalf, BSc; Anthony Harris, MSc; Prasuna Reddy, PhD; Philip G. Conaghan, PhD; Kim L. Bennell, PhD
\end{abstract}

IMPORTANCE There is debate about benefits of acupuncture for knee pain.

OBJECTIVE To determine the efficacy of laser and needle acupuncture for chronic knee pain.

DESIGN, SETTING, AND PARTICIPANTS Zelen-design clinical trial (randomization occurred before informed consent), in Victoria, Australia (February 2010-December 2012). Community volunteers ( 282 patients aged $\geq 50$ years with chronic knee pain) were treated by family physician acupuncturists.

INTERVENTIONS No acupuncture (control group, $n=71)$ and needle $(n=70)$, laser $(n=71)$, and sham laser $(n=70)$ acupuncture. Treatments were delivered for 12 weeks. Participants and acupuncturists were blinded to laser and sham laser acupuncture. Control participants were unaware of the trial.

MAIN OUTCOMES AND MEASURES Primary outcomes were average knee pain (numeric rating scale, 0 [no pain] to 10 [worst pain possible]; minimal clinically important difference [MCID], 1.8 units) and physical function (Western Ontario and McMaster Universities Osteoarthritis Index, 0 [no difficulty] to 68 [extreme difficulty]; MCID, 6 units) at 12 weeks. Secondary outcomes included other pain and function measures, quality of life, global change, and 1-year follow-up. Analyses were by intention-to-treat using multiple imputation for missing outcome data.

RESULTS At 12 weeks and 1 year, 26 (9\%) and 50 (18\%) participants were lost to follow-up, respectively. Analyses showed neither needle nor laser acupuncture significantly improved pain (mean difference; -0.4 units; $95 \% \mathrm{Cl},-1.2$ to 0.4 , and $-0.1 ; 95 \% \mathrm{Cl},-0.9$ to 0.7 , respectively) or function $(-1.7 ; 95 \% \mathrm{Cl},-6.1$ to 2.6 , and $0.5 ; 95 \% \mathrm{Cl},-3.4$ to 4.4 , respectively) compared with sham at 12 weeks. Compared with control, needle and laser acupuncture resulted in modest improvements in pain $(-1.1 ; 95 \% \mathrm{Cl},-1.8$ to -0.4 , and $-0.8 ; 95 \% \mathrm{Cl}$, -1.5 to -0.1 , respectively) at 12 weeks, but not at 1 year. Needle acupuncture resulted in modest improvement in function compared with control at 12 weeks $(-3.9 ; 95 \% \mathrm{Cl}$, -7.7 to -0.2$)$ but was not significantly different from sham $(-1.7 ; 95 \% \mathrm{Cl},-6.1$ to 2.6$)$ and was not maintained at 1 year. There were no differences for most secondary outcomes and no serious adverse events.

CONCLUSIONS AND RELEVANCE In patients older than 50 years with moderate or severe chronic knee pain, neither laser nor needle acupuncture conferred benefit over sham for pain or function. Our findings do not support acupuncture for these patients.

TRIAL REGISTRATION anzctr.org.au Identifier: ACTRN12609001001280
Author Audio Interview at jama.com

$\leftarrow$ Related article page 1342

+ Supplemental content at jama.com
Author Affiliations: Author affiliations are listed at the end of this article.

Corresponding Author: Rana S. Hinman, PhD, Centre for Health, Exercise and Sports Medicine, Department of Physiotherapy, School of Health Sciences, University of Melbourne, Victoria 3010, Australia (ranash@unimelb.edu.au). 
$\mathrm{C}$ hronic knee pain affects many people older than 50 years ${ }^{1}$ and is the most common pain concern among older people consulting family physicians. ${ }^{2}$ It is typically due to osteoarthritis and associated with declines in physical function. Nonpharmacological approaches are central to managing chronic knee pain. ${ }^{3,4}$ Patients with joint pain and arthritis frequently use complementary and alternative medicine. ${ }^{5}$ Acupuncture is the most popular of alternative medical systems, with use increasing over time. ${ }^{5}$ Although traditionally administered with needles, laser acupuncture (low-intensity laser therapy to acupuncture points) is a noninvasive alternative with evidence of benefit in some pain conditions. ${ }^{6-8}$ Efficacy of laser acupuncture compared with needles is unknown. Preliminary evidence suggests laser may be superior to needles for myofascial trigger points. ${ }^{9}$

A meta-analysis supports needle acupuncture for osteoarthritis pain. ${ }^{10}$ However, acupuncture effect sizes were much smaller when compared with sham needles rather than noacupuncture controls, and performance bias, response bias, or both may have influenced outcomes. Because patients have distinct acupuncture preconceptions, ${ }^{11}$ and positive attitudes toward acupuncture are consistently associated with significantly better treatment outcomes, ${ }^{12}$ recruitment bias may influence results from traditional clinical trials.

We conducted a Zelen-design clinical trial ${ }^{13}$ in which participants consented to acupuncture after randomization, and those randomized to receive no acupuncture (control group) were unaware of the clinical trial. Zelen designs can reduce risk of bias in a treatment trial in which knowledge of the intervention may influence recruitment (if only people with positive attitudes to the intervention seek to participate) and outcomes (where treatment expectations can inflate benefits, exacerbated by demoralization in untreated participants). Our aim was to determine efficacy of needle and laser acupuncture for chronic knee pain after 12 weeks and to evaluate maintenance of effects at 1 year. We hypothesized that laser acupuncture would be superior at 12 weeks compared with sham laser and needle acupuncture and that laser and needle acupuncture would be superior to no acupuncture.

\section{Methods}

A Zelen-design randomized clinical trial was conducted. The trial protocol ${ }^{14}$ adhered to STRICTA guidelines (see trial protocol in Supplement 1). ${ }^{15}$ The institutional human ethics committee approved the study. Participants provided written informed consent.

We recruited participants from metropolitan Melbourne and regional Victoria (Australia) via advertisements in the community, media, and medical/physical therapy clinics between February 2010 and December 2011. People were included if they were aged 50 years or older, had knee pain of longer than 3 months' duration, had knee pain most days with average severity of 4 or more out of 10 on a numeric rating scale (NRS), and had morning stiffness lasting less than
30 minutes. These criteria are consistent with clinical guidelines ${ }^{16}$ advocating a clinical diagnosis of osteoarthritis without need for investigations if these criteria are fulfilled. Exclusion criteria are provided in eTable 1 in Supplement 2. For bilaterally eligible knees, only the most symptomatic knee was evaluated (although acupuncturists were permitted to treat both knees).

\section{Randomization and Masking}

Participants initially consented to an observational study with repeated questionnaires over 1 year (Figure). A biostatistician (A.F.) prepared a randomization schedule (random permuted blocks of 6-12, stratified by acupuncturist). Participants who returned baseline questionnaires were consecutively randomized to receive laser acupuncture, sham laser acupuncture, ${ }^{17}$ needle acupuncture, or control (no acupuncture). An investigator (K.N.) accessed the computerized randomization schedule to reveal allocation.

Participants assigned to the control group continued in the observational study, unaware they were in an acupuncture trial and thus blinded. Other participants were invited to undergo their assigned intervention with those allocated to laser groups informed they could receive either active or sham laser acupuncture according to randomization. Participants in laser groups were thus blinded. Participants who accepted intervention provided further informed consent to participate. Participant codes for randomized laser treatment groups were preprogrammed into laser machines by an independent biomedical engineer to permit blinding of acupuncturists and participants in these groups. Participants receiving and acupuncturists delivering needle acupuncture were not blinded. Participants who declined acupuncture continued in the observational study.

\section{Interventions}

We used a combined Western and traditional Chinese medicine style of acupuncture. Eight family physicians registered as acupuncturists (mean, 33.3 years of clinical practice and 19.6 years of acupuncture experience ${ }^{14}$ ) delivered acupuncture. All were members of the Australian Medical Acupuncture College, had completed university-level acupuncture training, and were formally accredited (by examination and supervised clinical experience) and registered as medical practitioner acupuncturists by the Medical Board of Australia. Twentyminute treatments were delivered once or twice weekly for 12 weeks, with 8 to 12 sessions in total permitted.

Acupuncturists treated participants according to usual practice using a standardized set of acupuncture points (eTable 2 in Supplement 2), selecting from points around the knee as well as distal points. Other points could be used at the acupuncturist's discretion depending on clinical examination (eg, site and causes of pain). Initial treatment permitted a maximum of 6 points ( 4 on the study limb and 2 additional points chosen per protocol). In subsequent treatments, points were added and varied as clinically indicated. Single-use Seirin needles $(0.25 \times 40 \mathrm{~mm})$ were used for needle acupuncture (administered with the patient lying down and needles left in situ while the patient rested). Laser and sham laser acupunc- 


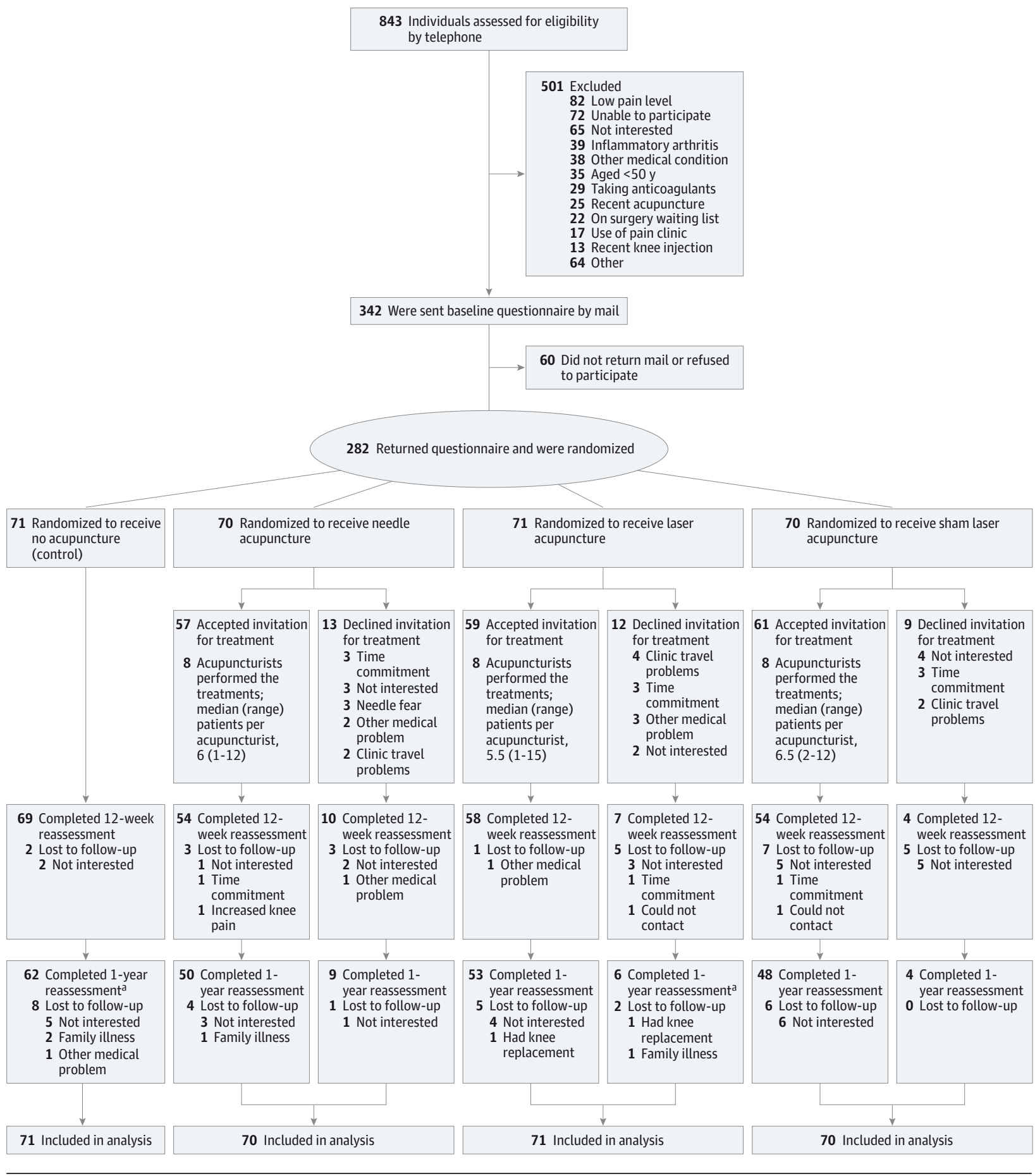

A participant was deemed lost to follow-up if all of the outcomes that were intended to be measured were missing at that follow-up. Participants lost to follow-up at 12 weeks were not followed up at 1 year, with single exceptions in each of the control and laser acupuncture groups.

${ }^{a}$ One rejoined at 1 year after having been lost to follow-up at 12 weeks.

ture were administered to selected points by custommanufactured Acupak (Melbourne) laser machines with the patient supine or sitting over the edge of a treatment couch. Standard Class 3B laser devices were used (measured output $10 \mathrm{~mW}$ and energy output $0.2 \mathrm{~J} /$ point, licensed by the Austra- lian Therapeutic Goods Administration), with a red nonlaser light at the probe tip that lit up in active and sham modes to maintain blinding. The red light level is below the threshold for measurable clinical effects, and previous research showed that 20 laser-familiar medical acupuncturists could not de- 


\begin{tabular}{|c|c|c|c|c|}
\hline Variable & $\begin{array}{l}\text { Control } \\
(n=71)\end{array}$ & $\begin{array}{c}\text { Needle } \\
(n=70)\end{array}$ & $\begin{array}{l}\text { Laser } \\
(n=71)\end{array}$ & $\begin{array}{c}\text { Sham Laser } \\
(n=70)\end{array}$ \\
\hline Age, mean (SD), y & $62.7(8.7)$ & $64.3(8.6)$ & $63.4(8.7)$ & $63.8(7.5)$ \\
\hline Female sex, №. (\%) & $40(56)$ & $32(46)$ & $28(39)$ & $39(56)$ \\
\hline Height, mean (SD), m & $1.70(0.11)$ & $1.71(0.10)$ & $1.71(0.10)$ & $1.71(0.10)$ \\
\hline Weight, mean (SD), kg & $85.6(20.8)$ & $86.3(17.7)$ & $89.3(20.2)$ & $84.7(19.3)$ \\
\hline Body mass index, mean (SD) ${ }^{a}$ & $29.3(5.8)$ & $29.8(5.8)$ & $30.7(6.1)$ & $28.8(5.4)$ \\
\hline \multicolumn{5}{|l|}{ Symptom duration, №. (\%) } \\
\hline$<1 \mathrm{y}$ & $6(8)$ & $2(3)$ & $12(17)$ & $4(6)$ \\
\hline 1 to $<5 y$ & $25(35)$ & $20(29)$ & $21(30)$ & $21(30)$ \\
\hline 5 to $<10 y$ & $18(25)$ & $18(26)$ & $10(14)$ & $10(14)$ \\
\hline$\geq 10 y$ & $19(27)$ & $29(41)$ & $27(38)$ & $35(50)$ \\
\hline Unilateral symptoms, №. (\%) & $35(49)$ & $25(36)$ & $24(34)$ & $26(37)$ \\
\hline \multicolumn{5}{|l|}{ Medication use, No. (\%) ${ }^{c}$} \\
\hline Analgesia & $34(48)$ & $28(40)$ & $27(38)$ & $37(53)$ \\
\hline NSAIDs & $14(20)$ & $14(20)$ & $14(20)$ & $16(23)$ \\
\hline COX-2 inhibitors & $3(4)$ & $6(9)$ & $6(8)$ & $3(4)$ \\
\hline Opioids & $1(1)$ & $1(1)$ & $2(3)$ & $1(1)$ \\
\hline Glucosamine products & $20(28)$ & $16(23)$ & $16(23)$ & $18(26)$ \\
\hline Fish oil & $20(28)$ & $18(26)$ & $13(18)$ & $20(29)$ \\
\hline \multicolumn{5}{|l|}{ Past treatment, No. (\%) ${ }^{\mathrm{d}}$} \\
\hline Physical therapy & $29(41)$ & $30(43)$ & $35(49)$ & $36(51)$ \\
\hline Surgery & $23(32)$ & $26(37)$ & $24(34)$ & 27 (39) \\
\hline Injections & $6(8)$ & $8(11)$ & $6(8)$ & $12(17)$ \\
\hline Acupuncture & $5(7)$ & $5(7)$ & $9(13)$ & $2(3)$ \\
\hline Exercise & $37(52)$ & $37(53)$ & $40(56)$ & $42(60)$ \\
\hline Hydrotherapy & $8(11)$ & $9(13)$ & $9(13)$ & $13(19)$ \\
\hline
\end{tabular}

\author{
Abbreviations: COX-2, \\ cyclooxygenase-2; NSAID, \\ nonsteroidal anti-inflammatory \\ drugs. \\ ${ }^{\text {a } C a l c u l a t e d ~ a s ~ w e i g h t ~ i n ~ k i l o g r a m s ~}$ \\ divided by height in meters \\ squared. \\ ${ }^{\mathrm{b}}$ Not all numbers add up to totals due \\ to some missing data. \\ ' Defined as medications purchased \\ over previous 4 weeks for knee \\ pain. \\ d Defined as treatments sought for \\ knee pain any time previously.
}

tect laser diode operation. ${ }^{18}$ Acupuncturists entered the participant code into the machine, which delivered active laser or sham laser according to the preprogrammed schedule. Acupuncturists completed standardized treatment notes.

\section{Outcome Measurements}

Participants completed questionnaires at baseline, 12 weeks, and 1 year. Primary outcomes were valid and reliable selfreported pain ${ }^{19}$ and function ${ }^{20}$ measures for osteoarthritis. Average knee pain over the previous week was measured using an 11-point NRS with terminal descriptors of "no pain" and "worst pain possible" (0-10, higher scores indicating worse knee pain; minimal clinically important difference [MCID], 1.8 units, extrapolated from 18-mm MCID reported for 100-mm visual analogue scales ${ }^{21}$ ). Physical function was measured using the Western and McMaster Universities Osteoarthritis Index (WOMAC, Likert version 3.1) function subscale, scored from o to 68 with higher scores indicating worse function (MCID, 6 nonnormalized units ${ }^{22}$ ).

Secondary outcomes included average knee pain on walking and standing, using the NRS (MCID, 1.8 units, extrapolated from 18-mm MCID reported for $100-\mathrm{mm}$ visual analogue scales ${ }^{21}$ ), average daily activity restriction over the previous week (NRS, 0-10, higher scores indicating more restriction), pain on the WOMAC pain subscale (0-20, higher scores indicating worse pain, MCID $\geq 12 \%$ improvement from baseline $^{23}$ ), health-related quality of life using the Assess- ment of Quality of Life instrument version 2 ( -0.04 to 1.00, higher scores indicating better quality of life, MCID, 0.06 units $^{24}$ ) and physical and mental component summary scores of the 12-item Short Form Health Survey (SF-12, 0-100, higher scores indicating better functioning, MCID $\geq 12 \%$ improvement from baseline ${ }^{23}$ ). At follow-up, participants rated global change overall, in pain, and in physical function using a 5-point ordinal scale (ranging from much worse to much better).

Acupuncturists recorded attendance. Adverse events were recorded by acupuncturists and open-ended participant questioning at 12 weeks. Use of health care (over previous month) was recorded in log books at baseline, 12 weeks, and 1 year to measure co-interventions. In the laser groups after first treatment, participants and acupuncturists indicated which treatment had been administered (active or sham) to evaluate blinding.

\section{Sample Size}

We aimed to detect an MCID of 1.8 units in NRS pain (0-10 scale, $100 \mathrm{~mm}$ in length and marked at $10-\mathrm{mm}$ increments). This was extrapolated from the conservative 17.5-mm MCID (rounded to $18 \mathrm{~mm}$ ) defined by expert consensus for osteoarthritis pain measurement on 0- to $100-\mathrm{mm}$ visual analogue scales. ${ }^{21} \mathrm{We}$ aimed to detect an MCID of 6 nonnormalized WOMAC physical function units (0-68 scale), extrapolated from the 9.1 normalized units (0-100 scale) identified as the MCID for osteoarthritis. ${ }^{22}$ Calculations were based on an analysis of covariance adjusting for baseline outcome scores, assuming 
Table 2. Mean (SD) Scores on Outcome Measures Over Time According to Group

\begin{tabular}{|c|c|c|c|c|c|c|c|c|c|c|c|c|}
\hline \multirow[b]{2}{*}{$\begin{array}{l}\text { Outcome } \\
\text { Measure }\end{array}$} & \multicolumn{4}{|c|}{ Baseline } & \multicolumn{4}{|c|}{12 Weeks } & \multicolumn{4}{|c|}{1 Year } \\
\hline & $\begin{array}{l}\text { Control } \\
(n=71)\end{array}$ & $\begin{array}{c}\begin{array}{c}\text { Needle } \\
(n=70)\end{array} \\
\end{array}$ & $\begin{array}{c}\begin{array}{c}\text { Laser } \\
(\mathrm{n}=71)\end{array} \\
\end{array}$ & $\begin{array}{c}\text { Sham } \\
\text { Laser } \\
(\mathrm{n}=70)\end{array}$ & $\begin{array}{l}\text { Control } \\
(n=69)\end{array}$ & $\begin{array}{c}\text { Needle } \\
(n=64)\end{array}$ & $\begin{array}{c}\text { Laser } \\
(\mathrm{n}=65)\end{array}$ & $\begin{array}{c}\text { Sham } \\
\text { Laser } \\
(\mathrm{n}=58)\end{array}$ & $\begin{array}{l}\text { Control } \\
(n=62)\end{array}$ & $\begin{array}{c}\text { Needle } \\
(n=59)\end{array}$ & $\begin{array}{c}\text { Laser } \\
(\mathrm{n}=58)\end{array}$ & $\begin{array}{c}\text { Sham } \\
\text { Laser } \\
(\mathrm{n}=51)\end{array}$ \\
\hline \multicolumn{13}{|l|}{ Primary } \\
\hline Overall pain ${ }^{a}$ & $\begin{array}{c}5.1 \\
(2.1)\end{array}$ & $\begin{array}{c}5.3 \\
(1.9)\end{array}$ & $\begin{array}{c}4.9 \\
(1.9)\end{array}$ & $\begin{array}{c}5.0 \\
(2.1)\end{array}$ & $\begin{array}{c}4.4 \\
(2.4)\end{array}$ & $\begin{array}{c}3.3 \\
(2.2)\end{array}$ & $\begin{array}{c}3.4 \\
(2.2)\end{array}$ & $\begin{array}{c}3.4 \\
(2.3)\end{array}$ & $\begin{array}{c}4.6 \\
(2.6)\end{array}$ & $\begin{array}{c}4.0 \\
(2.7)\end{array}$ & $\begin{array}{c}4.0 \\
(2.5)\end{array}$ & $\begin{array}{c}3.9 \\
(2.5)\end{array}$ \\
\hline $\begin{array}{l}\text { WOMAC } \\
\text { function }^{b}\end{array}$ & $\begin{array}{c}26.1 \\
(12.4)\end{array}$ & $\begin{array}{c}31.3 \\
(11.8)\end{array}$ & $\begin{array}{c}27.0 \\
(11.3)\end{array}$ & $\begin{array}{c}27.5 \\
(12.4)\end{array}$ & $\begin{array}{c}23.0 \\
(13.2)\end{array}$ & $\begin{array}{c}22.5 \\
(13.1)\end{array}$ & $\begin{array}{c}21.9 \\
(12.3)\end{array}$ & $\begin{array}{c}21.7 \\
(12.0)\end{array}$ & $\begin{array}{c}23.6 \\
(13.4)\end{array}$ & $\begin{array}{c}22.4 \\
(14.1)\end{array}$ & $\begin{array}{c}22.6 \\
(13.1)\end{array}$ & $\begin{array}{c}21.6 \\
(13.6)\end{array}$ \\
\hline \multicolumn{13}{|l|}{ Secondary } \\
\hline $\begin{array}{l}\text { Pain on } \\
\text { walking }^{\text {a }}\end{array}$ & $\begin{array}{c}4.8 \\
(2.1)\end{array}$ & $\begin{array}{l}5.5 \\
(2.0)\end{array}$ & $\begin{array}{c}4.8 \\
(2.0)\end{array}$ & $\begin{array}{c}5.2 \\
(2.2)\end{array}$ & $\begin{array}{c}4.3 \\
(2.4)\end{array}$ & $\begin{array}{c}3.4 \\
(2.2)\end{array}$ & $\begin{array}{c}3.6 \\
(2.4)\end{array}$ & $\begin{array}{c}3.7 \\
(2.6)\end{array}$ & $\begin{array}{c}4.4 \\
(2.6)\end{array}$ & $\begin{array}{c}4.1 \\
(2.9)\end{array}$ & $\begin{array}{c}4.1 \\
(2.6)\end{array}$ & $\begin{array}{c}4.2 \\
(2.6)\end{array}$ \\
\hline $\begin{array}{l}\text { Pain on } \\
\text { standing }\end{array}$ & $\begin{array}{c}4.1 \\
(2.4)\end{array}$ & $\begin{array}{c}4.6 \\
(2.2)\end{array}$ & $\begin{array}{c}3.8 \\
(2.1)\end{array}$ & $\begin{array}{c}4.3 \\
(2.3)\end{array}$ & $\begin{array}{c}3.8 \\
(2.5)\end{array}$ & $\begin{array}{c}3.2 \\
(2.3)\end{array}$ & $\begin{array}{c}3.3 \\
(2.4)\end{array}$ & $\begin{array}{c}2.9 \\
(2.4)\end{array}$ & $\begin{array}{c}4.0 \\
(2.6)\end{array}$ & $\begin{array}{c}3.7 \\
(2.9)\end{array}$ & $\begin{array}{c}3.8 \\
(2.6)\end{array}$ & $\begin{array}{c}3.5 \\
(2.9)\end{array}$ \\
\hline $\begin{array}{l}\text { Activity } \\
\text { restriction }{ }^{a}\end{array}$ & $\begin{array}{c}4.1 \\
(2.5)\end{array}$ & $\begin{array}{c}5.0 \\
(2.5)\end{array}$ & $\begin{array}{c}4.3 \\
(2.3)\end{array}$ & $\begin{array}{c}4.5 \\
(2.6)\end{array}$ & $\begin{array}{c}3.8 \\
(2.6)\end{array}$ & $\begin{array}{c}3.3 \\
(2.5)\end{array}$ & $\begin{array}{c}3.0 \\
(2.5)\end{array}$ & $\begin{array}{c}2.8 \\
(2.5)\end{array}$ & $\begin{array}{c}4.1 \\
(2.7)\end{array}$ & $\begin{array}{c}3.4 \\
(2.9)\end{array}$ & $\begin{array}{c}3.7 \\
(2.8)\end{array}$ & $\begin{array}{c}3.9 \\
(2.6)\end{array}$ \\
\hline WOMAC painc & $\begin{array}{c}7.8 \\
(3.4)\end{array}$ & $\begin{array}{c}9.0 \\
(3.3)\end{array}$ & $\begin{array}{c}8.3 \\
(3.1)\end{array}$ & $\begin{array}{c}8.6 \\
(3.5)\end{array}$ & $\begin{array}{c}7.3 \\
(3.9)\end{array}$ & $\begin{array}{c}6.7 \\
(3.8)\end{array}$ & $\begin{array}{c}6.6 \\
(3.9)\end{array}$ & $\begin{array}{c}6.6 \\
(3.9)\end{array}$ & $\begin{array}{c}7.4 \\
(4.1)\end{array}$ & $\begin{array}{c}6.7 \\
(4.0)\end{array}$ & $\begin{array}{c}7.1 \\
(4.1)\end{array}$ & $\begin{array}{c}6.9 \\
(4.0)\end{array}$ \\
\hline$A Q \circ L-6 D^{d}$ & $\begin{array}{c}0.77 \\
(0.16)\end{array}$ & $\begin{array}{c}0.72 \\
(0.15)\end{array}$ & $\begin{array}{c}0.70 \\
(0.16)\end{array}$ & $\begin{array}{c}0.73 \\
(0.15)\end{array}$ & $\begin{array}{c}0.79 \\
(0.16)\end{array}$ & $\begin{array}{c}0.75 \\
(0.18)\end{array}$ & $\begin{array}{c}0.73 \\
(0.17)\end{array}$ & $\begin{array}{c}0.78 \\
(0.12)\end{array}$ & $\begin{array}{c}0.77 \\
(0.16)\end{array}$ & $\begin{array}{c}0.74 \\
(0.17)\end{array}$ & $\begin{array}{c}0.73 \\
(0.17)\end{array}$ & $\begin{array}{c}0.74 \\
(0.16)\end{array}$ \\
\hline SF-12 PCSe & $\begin{array}{l}39.2 \\
(9.0)\end{array}$ & $\begin{array}{l}36.6 \\
(9.0)\end{array}$ & $\begin{array}{c}37.6 \\
(10.3)\end{array}$ & $\begin{array}{l}37.9 \\
(9.6)\end{array}$ & $\begin{array}{c}39.5 \\
(10.7)\end{array}$ & $\begin{array}{l}40.7 \\
(9.6)\end{array}$ & $\begin{array}{l}39.4 \\
(9.5)\end{array}$ & $\begin{array}{c}40.2 \\
(10.1)\end{array}$ & $\begin{array}{c}38.9 \\
(11.2)\end{array}$ & $\begin{array}{c}41.7 \\
(10.8)\end{array}$ & $\begin{array}{c}38.8 \\
(10.2)\end{array}$ & $\begin{array}{l}38.2 \\
(9.9)\end{array}$ \\
\hline SF-12 MCS & $\begin{array}{c}55.6 \\
(10.2)\end{array}$ & $\begin{array}{c}51.3 \\
(11.4)\end{array}$ & $\begin{array}{c}52.5 \\
(11.1)\end{array}$ & $\begin{array}{l}52.4 \\
(9.5)\end{array}$ & $\begin{array}{l}55.8 \\
(9.1)\end{array}$ & $\begin{array}{c}51.5 \\
(11.0)\end{array}$ & $\begin{array}{l}53.0 \\
(9.9)\end{array}$ & $\begin{array}{c}53.2 \\
(10.4)\end{array}$ & $\begin{array}{c}54.4 \\
(10.2)\end{array}$ & $\begin{array}{c}51.1 \\
(11.0)\end{array}$ & $\begin{array}{l}52.1 \\
(9.8)\end{array}$ & $\begin{array}{l}52.8 \\
(9.1)\end{array}$ \\
\hline
\end{tabular}

Abbreviations: AQoL-6D, Assessment of Quality of Life instrument version 2; SF-12, 12-item Short Form Health Survey; MCS, mental component summary; PCS, physical component summary; WOMAC, Western Ontario and McMaster Universities Osteoarthritis Index.

a Numeric rating scale range, 0-10; higher scores indicate worse pain/activity restriction. Minimal clinically important difference was 1.8 units for pain.

physical function. Minimal clinically important difference was 6 nonnormalized units.

' WOMAC pain subscale range, 0-20; higher scores indicate worse pain.

${ }^{d}$ Range, -0.04 to 1.00; higher scores indicate better quality of life.

e Range, 0-100; higher scores indicate better health.

${ }^{\mathrm{b}}$ WOMAC physical function subscale range, 0-68; higher scores indicate worse

between-patient SDs of 3.0 units for pain and 12.0 units for function, conservative intratherapist correlation of $0.10,15 \%$ nonconsent rate for participants randomized to an intervention group, and $15 \%$ attrition rate. ${ }^{14}$ To achieve $80 \%$ power at a 2-sided 5\% significance level, 66 patients were required in each group, which we rounded up to 70 .

\section{Statistical Analysis}

Analysis was by blinded biostatisticians (A.F. and E.W.) using Stata (version 13.1) software (StataCorp). We used an intention-to-treat approach with an analysis with missing data imputed using chained equations with predictive mean matching, imputing data for each group separately. Estimates from 20 imputed datasets were combined using Rubin rules. A complete case analysis including all available data was also used. $P$ values less than .05 were considered statistically significant and tests were 2-sided. We performed between-group comparisons of each acupuncture group with control, as well as pairwise comparisons between the 3 acupuncture groups. Differences in mean changes from baseline for each outcome at each time were compared between groups using mixed-effects linear regression modeling adjusting for baseline outcome scores. For intervention groups, acupuncturist was included as a random effect to account for clustering by acupuncturist. Global change ratings were dichotomized as improved (much better and slightly better) or not (unchanged to much worse) and compared between groups using mixed-effects logistic regression with acupuncturist as a random effect.
Secondary analyses addressed potential dilution effects due to the Zelen design. We performed analyses "as treated," which compared participants by treatment received regardless of randomization. However, such analyses are biased if participants who adhere to their assigned treatment differ from those who do not. ${ }^{25}$ We therefore also used a 2-stage least squares approach to estimate acupuncture effects under hypothetical full adherence to the randomized intervention (defined as 8 acupuncture sessions). This involved fitting a regression model for participation (the number of sessions attended) for each noncontrol treatment, and a second regression model predicting the outcome given participation, fitting all models simultaneously to account for all model uncertainty within the estimated standard errors. ${ }^{25}$ In this latter analysis, randomization acts as an instrumental variable, providing some protection against the biases of the as-treated analysis.

\section{Results}

Of 843 volunteers, 561 (67\%) were ineligible, refused to participate, or failed to return questionnaires (Figure). Thus, 282 participants were enrolled. Slightly fewer allocated to receive sham laser acupuncture declined treatment (9/70, $13 \%)$ compared with needle (13/70, 19\%) and laser acupuncture $(12 / 71,17 \%)$. At 12 weeks and 1 year, 26 (9\%) and 50 (18\%) participants were lost to follow-up, respectively. Groups were similar at baseline (Table 1), except sham laser 
Table 3. Estimated Mean Differences Between Groups, Adjusted for the Baseline Value of the Measure, at 12 Weeks With Multiple Imputation for Missing Data

\begin{tabular}{|c|c|c|c|c|c|c|c|c|c|c|c|c|}
\hline & \multicolumn{6}{|c|}{ Compared With Control Group } & \multicolumn{6}{|c|}{ Other Planned Contrasts } \\
\hline & \multicolumn{2}{|c|}{ Needles } & \multicolumn{2}{|c|}{ Laser } & \multicolumn{2}{|c|}{ Sham Laser } & \multicolumn{2}{|c|}{ Needles vs Laser } & \multicolumn{2}{|c|}{$\begin{array}{c}\text { Needles vs Sham } \\
\text { Laser }\end{array}$} & \multicolumn{2}{|c|}{ Laser vs Sham Laser } \\
\hline & $\begin{array}{l}\text { Estimate } \\
(95 \% \mathrm{Cl})\end{array}$ & $\begin{array}{c}P \\
\text { Value }\end{array}$ & $\begin{array}{l}\text { Estimate } \\
(95 \% \mathrm{Cl})\end{array}$ & $\begin{array}{c}P \\
\text { Value }\end{array}$ & $\begin{array}{l}\text { Estimate } \\
(95 \% \mathrm{Cl})\end{array}$ & $\begin{array}{c}P \\
\text { Value }\end{array}$ & $\begin{array}{l}\text { Estimate } \\
(95 \% \mathrm{Cl})\end{array}$ & $\begin{array}{c}P \\
\text { Value }\end{array}$ & $\begin{array}{l}\text { Estimate } \\
(95 \% \mathrm{Cl})\end{array}$ & $\begin{array}{c}P \\
\text { Value }\end{array}$ & $\begin{array}{l}\text { Estimate } \\
(95 \% \mathrm{Cl})\end{array}$ & $\begin{array}{c}P \\
\text { Value }\end{array}$ \\
\hline \multicolumn{13}{|l|}{ Primary } \\
\hline $\begin{array}{l}\text { Overall } \\
\text { pain }^{a, b}\end{array}$ & $\begin{array}{c}-1.1 \\
(-1.8 \text { to } \\
-0.4)\end{array}$ & .002 & $\begin{array}{c}-0.8 \\
(-1.5 \text { to } \\
-0.1)\end{array}$ & .03 & $\begin{array}{c}-0.7 \\
(-1.5 \text { to } \\
0.1)\end{array}$ & .07 & $\begin{array}{c}-0.3 \\
(-1.0 \text { to } \\
0.4)\end{array}$ & .38 & $\begin{array}{c}-0.4 \\
(-1.2 \text { to } \\
0.4)\end{array}$ & .34 & $\begin{array}{c}-0.1 \\
(-0.9 \text { to } \\
0.7)\end{array}$ & .86 \\
\hline $\begin{array}{l}\text { WOMAC } \\
\text { function }^{\mathrm{a}, \mathrm{c}}\end{array}$ & $\begin{array}{c}-3.9 \\
(-7.7 \text { to } \\
-0.2)\end{array}$ & .04 & $\begin{array}{c}-1.7 \\
(-5.1 \text { to } \\
1.8)\end{array}$ & .34 & $\begin{array}{c}-2.2 \\
(-6.1 \text { to } \\
1.8)\end{array}$ & .28 & $\begin{array}{c}-2.3 \\
(-6.1 \text { to } \\
1.6)\end{array}$ & .25 & $\begin{array}{c}-1.7 \\
(-6.1 \text { to } \\
2.6)\end{array}$ & .43 & $\begin{array}{c}0.5 \\
(-3.4 \text { to } \\
4.4)\end{array}$ & .79 \\
\hline \multicolumn{13}{|l|}{ Secondary } \\
\hline $\begin{array}{l}\text { Pain on } \\
\text { walking }\end{array}$ & $\begin{array}{c}-1.2 \\
(-2.0 \text { to } \\
-0.4)\end{array}$ & .003 & $\begin{array}{c}-0.6 \\
(-1.4 \text { to } \\
0.2)\end{array}$ & .16 & $\begin{array}{c}-0.7 \\
(-1.6 \text { to } \\
0.2)\end{array}$ & .15 & $\begin{array}{c}-0.6 \\
(-1.4 \text { to } \\
0.1)\end{array}$ & .11 & $\begin{array}{c}-0.5 \\
(-1.4 \text { to } \\
0.3)\end{array}$ & .22 & $\begin{array}{c}0.1 \\
(-0.8 \text { to } \\
1.0)\end{array}$ & .84 \\
\hline $\begin{array}{l}\text { Pain on } \\
\text { standing }\end{array}$ & $\begin{array}{c}-0.8 \\
(-1.5 \text { to } \\
0.0)\end{array}$ & .05 & $\begin{array}{c}-0.4 \\
(-1.2 \text { to } \\
0.4)\end{array}$ & .31 & $\begin{array}{c}-0.8 \\
(-1.6 \text { to } \\
0.0)\end{array}$ & .06 & $\begin{array}{c}-0.4 \\
(-1.2 \text { to } \\
0.4)\end{array}$ & .36 & $\begin{array}{c}0.0 \\
(-0.8 \text { to } \\
0.8)\end{array}$ & .93 & $\begin{array}{c}0.4 \\
(-0.5 \text { to } \\
1.3)\end{array}$ & .37 \\
\hline $\begin{array}{l}\text { Activity } \\
\text { restriction }{ }^{\mathrm{a}, \mathrm{b}}\end{array}$ & $\begin{array}{c}-0.8 \\
(-1.7 \text { to } \\
0.1)\end{array}$ & .08 & $\begin{array}{c}-0.8 \\
(-1.7 \text { to } \\
0.1)\end{array}$ & .09 & $\begin{array}{c}-0.9 \\
(-1.9 \text { to } \\
0.1)\end{array}$ & .07 & $\begin{array}{c}0.0 \\
(-0.8 \text { to } \\
0.9)\end{array}$ & .99 & $\begin{array}{c}0.1 \\
(-0.8 \text { to } \\
1.1)\end{array}$ & .78 & $\begin{array}{c}0.1 \\
(-0.8 \text { to } \\
1.1)\end{array}$ & .80 \\
\hline $\begin{array}{l}\text { WOMAC } \\
\text { pain }^{a, d}\end{array}$ & $\begin{array}{c}-1.2 \\
(-2.3 \text { to } \\
0.0)\end{array}$ & .05 & $\begin{array}{c}-1.0 \\
(-2.1 \text { to } \\
0.2)\end{array}$ & .10 & $\begin{array}{c}-1.0 \\
(-2.3 \text { to } \\
0.3)\end{array}$ & .12 & $\begin{array}{c}-0.2 \\
(-1.4 \text { to } \\
1.0)\end{array}$ & .76 & $\begin{array}{c}-0.1 \\
(-1.4 \text { to } \\
1.1)\end{array}$ & .83 & $\begin{array}{c}0.1 \\
(-1.3 \text { to } \\
1.4)\end{array}$ & .93 \\
\hline$A Q o L-6 D^{e, f}$ & $\begin{array}{c}-0.01 \\
(-0.06 \text { to } \\
0.04)\end{array}$ & .71 & $\begin{array}{c}0.00 \\
(-0.05 \text { to } \\
0.04)\end{array}$ & .87 & $\begin{array}{c}0.00 \\
(-0.04 \text { to } \\
0.05)\end{array}$ & .87 & $\begin{array}{c}-0.01 \\
(-0.06 \text { to } \\
0.05)\end{array}$ & .84 & $\begin{array}{c}-0.01 \\
(-0.07 \text { to } \\
0.04)\end{array}$ & .64 & $\begin{array}{c}-0.01 \\
(-0.06 \text { to } \\
0.05)\end{array}$ & .78 \\
\hline $\begin{array}{l}\text { SF-12 } \\
\mathrm{PCS}^{\mathrm{e}, g}\end{array}$ & $\begin{array}{c}2.7 \\
(-0.5 \text { to } \\
5.9)\end{array}$ & .10 & $\begin{array}{c}0.8 \\
(-2.4 \text { to } \\
4.0)\end{array}$ & .62 & $\begin{array}{c}0.9 \\
(-2.9 \text { to } \\
4.7)\end{array}$ & .65 & $\begin{array}{c}1.9 \\
(-1.4 \text { to } \\
5.1)\end{array}$ & .26 & $\begin{array}{c}1.8 \\
(-2.0 \text { to } \\
5.6)\end{array}$ & .35 & $\begin{array}{c}-0.1 \\
(-4.1 \text { to } \\
3.9)\end{array}$ & .97 \\
\hline $\begin{array}{l}\text { SF-12 } \\
\text { MCS }^{e, g}\end{array}$ & $\begin{array}{c}-2.4 \\
(-5.9 \text { to } \\
1.2)\end{array}$ & .19 & $\begin{array}{c}-1.7 \\
(-5.0 \text { to } \\
1.6)\end{array}$ & .32 & $\begin{array}{c}-1.3 \\
(-5.1 \text { to } \\
2.5)\end{array}$ & .50 & $\begin{array}{c}-0.7 \\
(-4.0 \text { to } \\
2.5)\end{array}$ & .67 & $\begin{array}{c}-1.1 \\
(-4.8 \text { to } \\
2.7)\end{array}$ & .57 & $\begin{array}{c}-0.4 \\
(-3.9 \text { to } \\
3.2)\end{array}$ & .83 \\
\hline
\end{tabular}

Abbreviations: AQoL-6D, Assessment of Quality of Life instrument version 2; MCS, mental component summary; PCS, physical component summary; SF-12, 12-item Short Form Health Survey; WOMAC, Western Ontario and McMaster Universities Osteoarthritis Index.

${ }^{\text {a }}$ Negative values indicate better (adjusted) mean in that (first-named) group compared with comparison group.

${ }^{\mathrm{b}}$ Numeric rating scale range, 0-10; higher scores indicate worse pain/activity restriction. Minimal clinically important difference was 1.8 units for pain.

worse physical function. Minimal clinically important difference was 6 nonnormalized units.

${ }^{d}$ WOMAC pain subscale range, 0-20; higher scores indicate worse pain.

e Positive values indicate better (adjusted) mean in that (first-named) group compared with comparison group.

${ }^{f}$ Range, -0.04 to 1.00; higher scores indicate better quality of life.

${ }^{g}$ Range, 0-100; higher scores indicate better health.

' WOMAC physical function subscale range, 0-68; higher scores indicate

had slightly more participants with symptoms exceeding 10 years' duration. Most participants attended 8 or more treatments (eTable 3 in Supplement 2). Adverse events were few, mild, and transient, and co-interventions were similar across groups (eTable 3). In the laser groups, most participants and acupuncturists were unable to identify whether active or sham acupuncture was delivered (eTable 4 in Supplement 2).

Table 2 summarizes continuous outcomes. There were no significant differences in primary outcomes between active and sham acupuncture at 12 weeks (Table 3) or 1 year (Table 4). Both needle and laser acupuncture resulted in modest improvements in pain compared with control at 12 weeks (Table 3 ) that were not maintained at 1 year (Table 4). Needle acupuncture improved physical function at 12 weeks compared with control but was not different from sham acupuncture (Table 3) and was not maintained at 1 year (Table 4). Most secondary outcomes showed no difference. Needle acupuncture improved pain on walking at 12 weeks (Table 3 ) but was not maintained at 1 year and improved activity restriction at 1 year (Table 4) compared with control. Complete case analyses gave similar results (eTables 5 and 6 in Supplement 2) with no differences persisting for primary outcomes between active and sham acupuncture. When analyzed as treated, there were still no differences in primary outcomes between active and sham acupuncture (eTables 7 and 8 in Supplement 2). Results changed negligibly under a model of full adherence to the randomized intervention (eTables 9 and 10 in Supplement 2). Similar proportions of participants in the acupuncture groups reported global improvements compared with control at 12 weeks and 1 year (eTable 11 in Supplement 2). Odds ratios for improvement were similar (eTable 11).

\section{Discussion}

Needle and laser acupuncture were no more efficacious than sham laser acupuncture. Although needle and laser 
Table 4. Estimated Mean Differences Between Groups, Adjusted for the Baseline Value of the Measure, at 1 Year With Multiple Imputation for Missing Data

\begin{tabular}{|c|c|c|c|c|c|c|c|c|c|c|c|c|}
\hline & \multicolumn{6}{|c|}{ Compared With Control Group } & \multicolumn{6}{|c|}{ Other Planned Contrasts } \\
\hline & \multicolumn{2}{|c|}{ Needles } & \multicolumn{2}{|c|}{ Laser } & \multicolumn{2}{|c|}{ Sham Laser } & \multicolumn{2}{|c|}{ Needles vs Laser } & \multicolumn{2}{|c|}{$\begin{array}{c}\text { Needles vs Sham } \\
\text { Laser }\end{array}$} & \multicolumn{2}{|c|}{ Laser vs Sham Laser } \\
\hline & $\begin{array}{l}\text { Estimate } \\
(95 \% \mathrm{CI})\end{array}$ & $\begin{array}{c}P \\
\text { Value }\end{array}$ & $\begin{array}{l}\text { Estimate } \\
(95 \% \mathrm{CI})\end{array}$ & $\begin{array}{c}P \\
\text { Value }\end{array}$ & $\begin{array}{l}\text { Estimate } \\
(95 \% \mathrm{Cl})\end{array}$ & $\begin{array}{c}P \\
\text { Value }\end{array}$ & $\begin{array}{l}\text { Estimate } \\
(95 \% \mathrm{Cl})\end{array}$ & $\begin{array}{c}P \\
\text { Value }\end{array}$ & $\begin{array}{l}\text { Estimate } \\
(95 \% \mathrm{Cl})\end{array}$ & $\begin{array}{c}P \\
\text { Value }\end{array}$ & $\begin{array}{l}\text { Estimate } \\
(95 \% \mathrm{Cl})\end{array}$ & $\begin{array}{c}P \\
\text { Value }\end{array}$ \\
\hline \multicolumn{13}{|l|}{ Primary } \\
\hline $\begin{array}{l}\text { Overall } \\
\text { pain }^{a, b}\end{array}$ & $\begin{array}{c}-0.7 \\
(-1.6 \text { to } \\
0.2)\end{array}$ & .14 & $\begin{array}{c}-0.6 \\
(-1.5 \text { to } \\
0.3)\end{array}$ & .19 & $\begin{array}{c}-0.6 \\
(-1.6 \text { to } \\
0.4)\end{array}$ & .22 & $\begin{array}{c}-0.1 \\
(-1.0 \text { to } \\
0.8)\end{array}$ & .83 & $\begin{array}{c}-0.1 \\
(-1.1 \text { to } \\
1.0)\end{array}$ & .91 & $\begin{array}{c}0.0 \\
(-0.9 \text { to } \\
1.0)\end{array}$ & .94 \\
\hline $\begin{array}{l}\text { WOMAC } \\
\text { function }{ }^{\mathrm{a}, \mathrm{c}}\end{array}$ & $\begin{array}{c}-3.7 \\
(-8.2 \text { to } \\
0.8)\end{array}$ & .11 & $\begin{array}{c}-0.6 \\
(-5.0 \text { to } \\
3.8)\end{array}$ & .80 & $\begin{array}{c}-1.7 \\
(-7.5 \text { to } \\
4.2)\end{array}$ & .57 & $\begin{array}{c}-3.1 \\
(-7.7 \text { to } \\
1.5)\end{array}$ & .19 & $\begin{array}{c}-2.0 \\
(-7.7 \text { to } \\
3.7)\end{array}$ & .49 & $\begin{array}{c}1.1 \\
(-4.8 \text { to } \\
7.0)\end{array}$ & .71 \\
\hline \multicolumn{13}{|l|}{ Secondary } \\
\hline $\begin{array}{l}\text { Pain on } \\
\text { walking }\end{array}$ & $\begin{array}{c}-0.6 \\
(-1.5 \text { to } \\
0.4)\end{array}$ & .27 & $\begin{array}{c}-0.3 \\
(-1.2 \text { to } \\
0.7)\end{array}$ & .57 & $\begin{array}{c}-0.3 \\
(-1.3 \text { to } \\
0.7)\end{array}$ & .56 & $\begin{array}{c}-0.3 \\
(-1.3 \text { to } \\
0.7)\end{array}$ & .59 & $\begin{array}{c}-0.2 \\
(-1.3 \text { to } \\
0.8)\end{array}$ & .65 & $\begin{array}{c}0.0 \\
(-1.0 \text { to } \\
1.1)\end{array}$ & .94 \\
\hline $\begin{array}{l}\text { Pain on } \\
\text { standing }\end{array}$ & $\begin{array}{c}-0.5 \\
(-1.4 \text { to } \\
0.5)\end{array}$ & .35 & $\begin{array}{c}-0.2 \\
(-1.1 \text { to } \\
0.8)\end{array}$ & .75 & $\begin{array}{c}-0.6 \\
(-1.7 \text { to } \\
0.5)\end{array}$ & .28 & $\begin{array}{c}-0.3 \\
(-1.3 \text { to } \\
0.7)\end{array}$ & .56 & $\begin{array}{c}0.2 \\
(-1.0 \text { to } \\
1.3)\end{array}$ & .79 & $\begin{array}{c}0.5 \\
(-0.7 \text { to } \\
1.6)\end{array}$ & .42 \\
\hline $\begin{array}{l}\text { Activity } \\
\text { restriction }{ }^{a, b}\end{array}$ & $\begin{array}{c}-1.1 \\
(-2.1 \text { to } \\
-0.2)\end{array}$ & .02 & $\begin{array}{c}-0.4 \\
(-1.4 \text { to } \\
0.5)\end{array}$ & .37 & $\begin{array}{c}-0.4 \\
(-1.4 \text { to } \\
0.6)\end{array}$ & .45 & $\begin{array}{c}-0.7 \\
(-1.7 \text { to } \\
0.3)\end{array}$ & .15 & $\begin{array}{c}-0.8 \\
(-1.8 \text { to } \\
0.3)\end{array}$ & .16 & $\begin{array}{c}-0.1 \\
(-1.1 \text { to } \\
1.0)\end{array}$ & .93 \\
\hline $\begin{array}{l}\text { WOMAC } \\
\text { pain }\end{array}$ & $\begin{array}{c}-1.4 \\
(-2.7 \text { to } \\
0.0)\end{array}$ & .05 & $\begin{array}{c}-0.4 \\
(-1.8 \text { to } \\
1.0)\end{array}$ & .59 & $\begin{array}{c}-0.3 \\
(-2.2 \text { to } \\
1.6)\end{array}$ & .72 & $\begin{array}{c}-1.0 \\
(-2.5 \text { to } \\
0.5)\end{array}$ & .18 & $\begin{array}{c}-1.0 \\
(-3.0 \text { to } \\
0.9)\end{array}$ & .29 & $\begin{array}{c}0.0 \\
(-1.9 \text { to } \\
1.9)\end{array}$ & .96 \\
\hline$A Q o L-6 D^{e, f}$ & $\begin{array}{c}-0.01 \\
(-0.07 \text { to } \\
0.05)\end{array}$ & .67 & $\begin{array}{c}0.01 \\
(-0.05 \text { to } \\
0.06)\end{array}$ & .80 & $\begin{array}{c}-0.01 \\
(-0.08 \text { to } \\
0.07)\end{array}$ & .87 & $\begin{array}{c}-0.02 \\
(-0.08 \text { to } \\
0.04)\end{array}$ & .50 & $\begin{array}{c}-0.01 \\
(-0.07 \text { to } \\
0.06)\end{array}$ & .83 & $\begin{array}{c}0.01 \\
(-0.05 \text { to } \\
0.08)\end{array}$ & .70 \\
\hline $\begin{array}{l}\text { SF-12 } \\
\mathrm{PCS}^{\mathrm{e}, \mathrm{g}}\end{array}$ & $\begin{array}{c}2.3 \\
(-1.7 \text { to } \\
6.3)\end{array}$ & .26 & $\begin{array}{c}-0.4 \\
(-4.4 \text { to } \\
3.6)\end{array}$ & .86 & $\begin{array}{c}-0.7 \\
(-5.0 \text { to } \\
3.5)\end{array}$ & .74 & $\begin{array}{c}2.7 \\
(-1.1 \text { to } \\
6.4)\end{array}$ & .17 & $\begin{array}{c}3.0 \\
(-1.4 \text { to } \\
7.4)\end{array}$ & .18 & $\begin{array}{c}0.4 \\
(-3.8 \text { to } \\
4.5)\end{array}$ & .86 \\
\hline $\begin{array}{l}\text { SF-12 } \\
\text { MCS }^{\mathrm{e}, \mathrm{g}}\end{array}$ & $\begin{array}{c}-0.9 \\
(-5.2 \text { to } \\
3.4)\end{array}$ & .67 & $\begin{array}{c}-0.9 \\
(-5.5 \text { to } \\
3.7)\end{array}$ & .69 & $\begin{array}{c}-0.3 \\
(-5.8 \text { to } \\
5.1)\end{array}$ & .90 & $\begin{array}{c}0.0 \\
(-4.1 \text { to } \\
4.1)\end{array}$ & .99 & $\begin{array}{c}-0.6 \\
(-5.6 \text { to } \\
4.4)\end{array}$ & .81 & $\begin{array}{c}-0.6 \\
(-5.4 \text { to } \\
4.2)\end{array}$ & .81 \\
\hline
\end{tabular}

Abbreviations: AQoL-6D, Assessment of Quality of Life instrument version 2; MCS, mental component summary; PCS, physical component summary; SF-12, 12-item Short Form Health Survey; WOMAC, Western Ontario and McMaster Universities Osteoarthritis Index.

${ }^{a}$ Negative values indicate better (adjusted) mean in that (first-named) group compared with comparison group.

${ }^{\mathrm{b}}$ Numeric rating scale range, 0-10; higher scores indicate worse pain/activity restriction. Minimal clinically important difference was 1.8 units for pain.

worse physical function. Minimal clinically important difference was 6 nonnormalized units.

${ }^{d}$ WOMAC pain subscale range, 0-20; higher scores indicate worse pain.

e Positive values indicate better (adjusted) mean in that (first-named) group compared with comparison group.

${ }^{f}$ Range, -0.04 to 1.00; higher scores indicate better quality of life.

g Range, 0-100; higher scores indicate better health.

c WOMAC physical function subscale range, 0-68; higher scores indicate

acupuncture improved pain after treatment compared with control, improvements were not sustained at 1 year and were of a clinically unimportant magnitude. ${ }^{21,26}$ Improvement in WOMAC physical function with needle acupuncture relative to control at 12 weeks was of a clinically irrelevant magnitude ${ }^{22}$ and did not persist at 1 year. Furthermore, this improvement was not different from sham laser.

Our findings partially agree with a systematic review and individual patient data meta-analysis on acupuncture efficacy for osteoarthritis by Vickers et al, ${ }^{10}$ which showed moderate improvements in pain with needle acupuncture compared with no acupuncture and much smaller but statistically significant improvements compared with sham. Our sample size may have contributed to the nonsignificant findings between active and sham laser acupuncture. We powered our study for between-group differences based on MCIDs in primary outcomes. Although a larger sample may have detected statistically significant effects of active acu- puncture relative to sham, the clinical relevance of such differences would be questionable. Our observed betweengroup differences were smaller than the MCIDs, and 95\% confidence intervals indicated that the ranges of plausible between-group differences were unlikely to have included differences of any practical importance.

Clinical guidelines vary regarding acupuncture recommendations for knee osteoarthritis. The American College of Rheumatology conditionally recommends acupuncture for people with chronic moderate or severe osteoarthritis pain who are arthroplasty candidates. ${ }^{4}$ The Osteoarthritis Research Society International is "uncertain" regarding acupuncture. ${ }^{27}$ Initial propositions regarding acupuncture for osteoarthritis did not achieve expert panel consensus from the European League Against Rheumatism, and acupuncture failed to feature in their final recommendations. ${ }^{3}$ The American Academy of Orthopaedic Surgeons ${ }^{28}$ "cannot recommend using acupuncture" for knee osteoarthritis 
and the National Institute for Health and Care Excellence osteoarthritis clinical guidelines ${ }^{16}$ advise against acupuncture. Findings from our trial support these latter recommendations.

Our Zelen design overcame some limitations of previous acupuncture studies. Patient preferences for acupuncture influence treatment outcomes, ${ }^{11,12,29}$ and our design ensured we did not recruit only patients with positive acupuncture attitudes. Previous trials have not been able to blind participants who do not receive acupuncture. The Zelen design allowed us to blind our control participants, minimizing the risk of demoralization in our untreated patients. Although Zelen-design trials may be considered unethical, ${ }^{30}$ there are prior examples in the literature, ${ }^{31}$ including trials of physiotherapy ${ }^{32}$ and arthroscopy ${ }^{33}$ for knee osteoarthritis. Ethical approval required us to disclose at enrollment that deidentified data would be used in a larger study evaluating treatments (undisclosed) for knee osteoarthritis. Thus, all participants consented to their data being used in ways other than for the observational study they enrolled in.

Our trial had a low risk of recruitment bias. Contrary to the patients in the meta-analysis by Vickers et al, ${ }^{10}$ our participants did not knowingly volunteer for an acupuncture study. Our sample may have held less-positive expectations about acupuncture compared with previous research, which may explain the diluted acupuncture effects (relative to control) we observed. Our findings may not be generalizable to patients who hold positive beliefs about acupuncture.

Acupuncture outcomes may be attributed to characteristic (specific, physiological) and incidental (nonspecific, contextual, placebo) effects. ${ }^{34}$ Incidental factors such as treatment setting, patient expectations and attitudes (such as optimism), acupuncturist's confidence in treatment, and patient and acupuncturist interaction may influence outcomes. ${ }^{35}$ In our study, benefits of acupuncture were exclusively attributed to incidental effects, given the lack of significant differences between active acupuncture and sham treatment. Continuous subjective measures, such as pain and self-reported physical function, as used in our study, are particularly subject to placebo responses. ${ }^{36} \mathrm{We}$ may have observed significant differences between active and sham acupuncture had we included objective outcome measures.

A major strength of our study is its Zelen design. Other aspects of our study, such as the head-to-head comparison of needle and laser acupuncture, acupuncturist blinding in laser groups, and measurement of 1-year outcomes, are important additions to the acupuncture literature. However, we cannot extrapolate beyond the 1-year time period for acupuncture outcomes, and it is possible that there are benefits of treatment beyond 1 year that we did not capture. There is little research evaluating laser acupuncture for chronic knee pain. As such, no laser acupuncture studies were included in the meta-analysis by Vickers et al. ${ }^{10}$ We minimized potential for response and performance bias in the laser acupuncture groups by including a sham treatment. Lack of acupuncturist and participant blinding to needle acupuncture may have introduced treatment bias, response bias, or both in this group and may explain why needle acupuncture improved pain and function relative to control at 12 weeks (NRS and WOMAC).

Limitations include $13 \%$ to $19 \%$ of participants declining treatment. Although consistent with other Zelen-design trials (mean, $13.8 \%$ crossover rate reported in 58 studies $^{31}$ ), it may have diluted acupuncture effects. However, when analyzed as treated, primary outcomes did not differ between active and sham acupuncture. We lost $18 \%$ of participants to follow-up by 1 year; however, our multiple imputation analyses corrected for any bias assuming the missing outcomes were missing at random. Some participants did not fully adhere to treatment, but modeling results under the hypothetical scenario of full adherence to the randomized intervention did not alter outcomes. Although there is little evidence that acupuncture characteristics modify outcomes, ${ }^{37}$ our findings cannot be generalized to different acupuncture regimens. We did not perform $\mathrm{x}$-rays. Our findings likely only apply to patients with clinically diagnosed osteoarthritis and moderate or severe pain (23\% [64/282 participants] had baseline pain scores $\geq 7 / 10$ ) and may not be generalizable to end-stage radiographic disease. We did not include sham needle acupuncture because of the inability to blind acupuncturists, because of concerns that sham needling may exert somatosensory effects, ${ }^{38,39}$ and because it has previously been studied. ${ }^{10}$

\section{Conclusions}

Among patients older than 50 years with moderate to severe chronic knee pain, neither laser nor needle acupuncture conferred benefit over sham for pain or function. Our findings do not support acupuncture for these patients.

\section{ARTICLE INFORMATION}

Author Affiliations: Centre for Health, Exercise and Sports Medicine, Department of Physiotherapy, School of Health Sciences, Faculty of Medicine, Dentistry and Health Sciences, University of Melbourne, Melbourne, Australia (Hinman, McCrory, Metcalf, Bennell); Florey Institute of Neuroscience and Mental Health, Melbourne Brain Centre, Heidelberg, Melbourne, Australia (McCrory); Department of General Practice, School of Medicine, Faculty of Medicine, Dentistry and
Health Sciences, University of Melbourne, Melbourne, Australia (Pirotta, Relf, Kyriakides, Novy); Epidemiology and Preventative Medicine, Faculty of Medicine, Nursing and Health Sciences, Monash University, Melbourne, Australia (Forbes) Division of Physiotherapy, School of Health and Rehabilitation Sciences, University of Queensland Brisbane, Australia (Crossley); Farr Institute of Health Informatics Research, London, United Kingdom (Williamson); Department of Medical Statistics, London School of Hygiene and Tropical Medicine, London, United Kingdom (Williamson);
Centre for Health Economics, Faculty of Business and Economics, Monash University, Melbourne, Australia (Harris); School of Medicine and Public Health, Faculty of Health and Medicine, University of Newcastle, Callaghan, Newcastle, Australia (Reddy); Leeds Institute of Rheumatic and Musculoskeletal Medicine, University of Leeds and NIHR Leeds Musculoskeletal Biomedical Research Unit, Leeds, United Kingdom (Conaghan). 
Author Contributions: Dr Hinman had full access to all of the data in the study and takes responsibility for the integrity of the data and the accuracy of the data analysis.

Study concept and design: Hinman, McCrory, Pirotta, Relf, Forbes, Crossley, Harris, Reddy, Conaghan, Bennell.

Acquisition, analysis, or interpretation of data: Hinman, McCrory, Pirotta, Relf, Forbes, Williamson, Kyriakides, Novy, Metcalf, Harris, Reddy, Bennell. Drafting of the manuscript: Hinman, McCrory, Pirotta, Relf, Forbes, Kyriakides, Novy, Metcalf, Harris, Bennell.

Critical revision of the manuscript for important intellectual content: Hinman, McCrory, Pirotta, Relf, Forbes, Crossley, Williamson, Kyriakides, Novy, Metcalf, Harris, Reddy, Conaghan, Bennell. Statistical analysis: Forbes, Williamson, Metcalf, Reddy.

Obtained funding: Hinman, McCrory, Pirotta, Relf, Crossley, Reddy, Conaghan, Bennell. Administrative, technical, or material support: McCrory, Relf, Kyriakides, Novy, Metcalf.

Study supervision: Hinman, McCrory, Pirotta, Relf, Bennell.

Conflict of Interest Disclosures: All authors have completed and submitted the ICMJE Form for Disclosure of Potential Conflicts of Interest. Dr Hinman reported having received (along with the University of Melbourne) royalties from the sales of a shoe (Gel Melbourne OA) by Asics. Dr McCrory reported having served as a consultant to Horse Racing Association UK, Cogstate, and University of Otago in New Zealand; having received grants from the Victorian Government, International Rugby Board, Australian Football League Research Foundation, Royal Australasian College of Surgeons Glaxo Wellcome, Ipsen, Janssen-Cilag, Schering, Novartis, and Parke-Davis; having received book royalties from McGraw Hill; having received payment for developing educational presentation from Axon Sports; having owned stock or stock options with Symphony Healthcare and Raptor Compression Technology; and having received travel expenses from FIFA (Fédération Internationale de Football Association) International Olympic Committee, Horse Racing Association UK, American Academy of Neurology, and Aspetar Hospital in Qatar. Dr Bennell reported having received consultation fees from Physitrack and (along with the University of Melbourne) royalties from the sales of a shoe (Gel Melbourne $\mathrm{OA}$ ) by Asics. No other disclosures were reported.

Funding/Support: This trial was funded by the National Health and Medical Research Council (project 566783). Drs Hinman and Bennell are both funded in part by Australian Research Council Future Fellowships (FT130100175 and FT0991413, respectively). Dr McCrory is funded in part by a National Health and Medical Research Council Practitioner Fellowship (1026383). Dr Pirotta is funded in part by a National Health and Medical Research Council Career Development Fellowship (1050830). Dr Williamson was funded in part by a National Health and Medical Research Council grant (1004233)

Role of the Funder/Sponsor: The study sponsor had no role in the design and conduct of the study: collection, management, analysis, and interpretation of the data; preparation, review, or approval of the manuscript; and decision to submit the manuscript for publication.
Additional Contributions: The project acupuncturists who provided the acupuncture interventions were paid on a consultancy basis for treatments provided. The doctors who delivered the acupuncture treatments were lan Relf, MBBS, Department of General Practice, School of Medicine, Faculty of Medicine, Dentistry and Health Sciences, University of Melbourne, Melbourne, Australia; Paul Ghaie, MBBS, Department of General Practice, Monash University, Melbourne; Sandra Martin, MBBS, Laser Therapy Centre, Glen Waverly, Melbourne; Danny Traum, MBBS, Dr D. J. Traum Laser Acupuncture Clinic, Malvern, Melbourne; Paul Coughlan, MBBCh, Traralgon Medical Centre, Traralgon, Australia; Robert Meyer, MBBS, Lime Medical Clinic, Mildura, Australia; Malcolm MacDonald, MBBS, Barnard St Clinic, Bendigo, Australia; Tim Denton, MBBS, Surfcoast Medical Centre, Torquay, and Geelong GP Super Clinic, Belmont, Australia.

\section{REFERENCES}

1. Jinks C, Jordan K, Ong BN, Croft P. A brief screening tool for knee pain in primary care (KNEST): 2, results from a survey in the general population aged 50 and over. Rheumatology (Oxford). 2004:43(1):55-61.

2. Mäntyselkä $P$, Kumpusalo $E$, Ahonen $R$, et al. Pain as a reason to visit the doctor: a study in Finnish primary health care. Pain. 2001;89(2-3): 175-180.

3. Fernandes L, Hagen KB, Bijlsma JW, et al; European League Against Rheumatism (EULAR). EULAR recommendations for the non-pharmacological core management of hip and knee osteoarthritis. Ann Rheum Dis. 2013;72(7): 1125-1135

4. Hochberg MC, Altman RD, April KT, et al; American College of Rheumatology. American College of Rheumatology 2012 recommendations for the use of nonpharmacologic and pharmacologic therapies in osteoarthritis of the hand, hip, and knee. Arthritis Care Res (Hoboken) 2012;64(4):465-474.

5. Barnes PM, Bloom B, Nahin RL. Complementary and alternative medicine use among adults and children: United States, 2007. Natl Health Stat Report. 2008;12(12):1-23.

6. Baxter GD, Bleakley C, McDonough S. Clinical effectiveness of laser acupuncture: a systematic review. J Acupunct Meridian Stud. 2008;1(2):65-82.

7. Chow RT, Johnson MI, Lopes-Martins RA, Bjordal JM. Efficacy of low-level laser therapy in the management of neck pain: a systematic review and meta-analysis of randomised placebo or active-treatment controlled trials. Lancet. 2009; 374(9705):1897-1908

8. Yousefi-Nooraie R, Schonstein E, Heidari K, et al Low level laser therapy for nonspecific low-back pain. Cochrane Database Syst Rev. 2007;2(2): CD005107.

9. Ilbuldu E, Cakmak A, Disci R, Aydin R. Comparison of laser, dry needling, and placebo laser treatments in myofascial pain syndrome. Photomed Laser Surg. 2004;22(4):306-311.

10. Vickers AJ, Cronin AM, Maschino AC, et al; Acupuncture Trialists' Collaboration. Acupuncture for chronic pain: individual patient data meta-analysis. Arch Intern Med. 2012;172(19):14441453.
11. Bishop FL, Lewith GT. Patients' preconceptions of acupuncture: a qualitative study exploring the decisions patients make when seeking acupuncture. BMC Complement Altern Med. 2013; 13:102.

12. Linde $K$, Witt CM, Streng A, et al. The impact of patient expectations on outcomes in four randomized controlled trials of acupuncture in patients with chronic pain. Pain. 2007;128(3):264 271.

13. Zelen M. A new design for randomized clinical trials. N Engl J Med. 1979;300(22):1242-1245.

14. Hinman RS, McCrory P, Pirotta M, et al. Efficacy of acupuncture for chronic knee pain: protocol for a randomised controlled trial using a Zelen design. BMC Complement Altern Med. 2012;12:161.

15. MacPherson H, Altman DG, Hammerschlag $R$, et al; STRICTA Revision Group. Revised STandards for Reporting Interventions in Clinical Trials of Acupuncture (STRICTA): extending the CONSORT statement. PLoS Med. 2010;7(6):e1000261.

16. Osteoarthritis: care and management in adults [clinical guideline CG177]: methods, evidence and recommendations. National Institute for Health and Care Excellence. http://www.nice.org.uk/guidance /CG177. Accessed September 8, 2014.

17. Irnich D, Salih N, Offenbächer M, Fleckenstein J. Is sham laser a valid control for acupuncture trials? Evid Based Complement Alternat Med. 2011;2011: 485945

18. Relf $I$, Chow R, Pirotta M. Blinding techniques in randomized controlled trials of laser therapy: an overview and possible solution. Evid Based Complement Alternat Med. 2008;5(4):383-389.

19. Hawker GA, Mian S, Kendzerska T, French M Measures of adult pain: Visual Analog Scale for Pain (VAS Pain), Numeric Rating Scale for Pain (NRS Pain), McGill Pain Questionnaire (MPQ), Short-Form McGill Pain Questionnaire (SF-MPQ), Chronic Pain Grade Scale (CPGS), Short Form-36 Bodily Pain Scale (SF-36 BPS), and Measure of Intermittent and Constant Osteoarthritis Pain (ICOAP). Arthritis Care Res (Hoboken). 2011;63(suppl 11):S240-S252.

20. Bellamy N, Buchanan WW, Goldsmith $\mathrm{CH}_{\text {, }}$ Campbell J, Stitt LW. Validation study of WOMAC: a health status instrument for measuring clinically important patient relevant outcomes to antirheumatic drug therapy in patients with osteoarthritis of the hip or knee. J Rheumatol. 1988; 15(12):1833-1840.

21. Bellamy N, Carette S, Ford PM, et al. Osteoarthritis antirheumatic drug trials: III, setting the delta for clinical trials: results of a consensus development (Delphi) exercise. J Rheumatol. 1992; 19(3):451-457.

22. Tubach F, Ravaud P, Baron G, et al. Evaluation of clinically relevant changes in patient reported outcomes in knee and hip osteoarthritis: the minimal clinically important improvement. Ann Rheum Dis. 2005;64(1):29-33.

23. Angst F, Aeschlimann A, Stucki G. Smallest detectable and minimal clinically important differences of rehabilitation intervention with their implications for required sample sizes using WOMAC and SF-36 quality of life measurement instruments in patients with osteoarthritis of the lower extremities. Arthritis Rheum. 2001;45(4): 384-391. 
24. Hawthorne G, Osborne R. Population norms and meaningful differences for the Assessment of Quality of Life (AQoL) measure. Aust N Z J Public Health. 2005;29(2):136-142.

25. Stuart EA, Perry DF, Le HN, lalongo NS Estimating intervention effects of prevention programs: accounting for noncompliance. Prev Sci. 2008;9(4):288-298.

26. Farrar JT, Young JP Jr, LaMoreaux L, Werth JL, Poole RM. Clinical importance of changes in chronic pain intensity measured on an 11-point numerical pain rating scale. Pain. 2001;94(2):149-158.

27. McAlindon TE, Bannuru RR, Sullivan MC, et al. OARSI guidelines for the non-surgical management of knee osteoarthritis. Osteoarthritis Cartilage. 2014;22(3):363-388

28. Treatment of osteoarthritis of the knee evidence-based guideline, 2nd edition. American Academy of Orthopaedic Surgeons. http://www.aaos.org/research/guidelines /treatmentofOsteoarthritisoftheKneeGuideline.pdf. Accessed September 8, 2014

29. Sherman KJ, Cherkin DC, Ichikawa L, et al. Characteristics of patients with chronic back pain who benefit from acupuncture. BMC Musculoskelet Disord. 2009;10:114.

30. Consent: how informed? Lancet. 1984;1(8392) 1445-1447.

31. Adamson J, Cockayne S, Puffer S, Torgerson DJ, Review of randomised trials using the post-randomised consent (Zelen's) design. Contemp Clin Trials. 2006;27(4):305-319.

32. Quilty B, Tucker M, Campbell R, Dieppe P. Physiotherapy, including quadriceps exercises and patellar taping, for knee osteoarthritis with predominant patello-femoral joint involvement: randomized controlled trial. J Rheumatol. 2003;30 (6):1311-1317

33. Chang RW, Falconer J, Stulberg SD, Arnold WJ, Dyer AR. Prerandomization: an alternative to classic randomization: the effects on recruitment in a controlled trial of arthroscopy for osteoarthrosis of the knee. J Bone Joint Surg Am. 1990;72(10):14511455.

34. Witt CM, Schützler $L$. The gap between result from sham-controlled trials and trials using other controls in acupuncture research: the influence of context. Complement Ther Med. 2013;21(2):112-114.
35. Abhishek A, Doherty M. Mechanisms of the placebo response in pain in osteoarthritis. Osteoarthritis Cartilage. 2013;21(9):1229-1235.

36. Zhang W, Robertson J, Jones AC, Dieppe PA, Doherty $M$. The placebo effect and its determinants in osteoarthritis: meta-analysis of randomised controlled trials. Ann Rheum Dis. 2008;67(12):1716 1723

37. MacPherson $\mathrm{H}$, Maschino AC, Lewith G, Foster NE, Witt CM, Vickers AJ; Acupuncture Trialists' Collaboration. Characteristics of acupuncture treatment associated with outcome: an individual patient meta-analysis of 17,922 patients with chronic pain in randomised controlled trials. PLOS One. 2013;8(10):e77438

38. Huang W, Pach D, Napadow V, et al. Characterizing acupuncture stimuli using brain imaging with FMRI: a systematic review and meta-analysis of the literature. PLoS One. 2012;7(4): e32960.

39. Lund I, Lundeberg T. Are minimal, superficial or sham acupuncture procedures acceptable as inert placebo controls? Acupunct Med. 2006;24(1):13-15 